\title{
Salivary and serum levels of substance $P$, neurokinin $A$ and calcitonin gene related peptide in burning mouth syndrome
}

\author{
Vanja-Vucicevic Boras ${ }^{1}$, Neil-William Savage ${ }^{1}$, Vlaho Brailo ${ }^{2}$, Josip Lukac ${ }^{3}$, Maja Lukac ${ }^{3}$, Iva Z Alajbeg ${ }^{4}$ \\ ${ }^{1}$ Oral Medicine, The University of Queensland School of Dentistry, Brisbane, Australia \\ ${ }^{2}$ Department of Oral Medicine, School of Dentistry, University of Zagreb, Zagreb, Croatia \\ ${ }^{3}$ Department of Oncology and Nuclear Medicine, Clinical Hospital "Sr. Milosrdnica", Zagreb, Croatia \\ ${ }^{4}$ Department of Prosthodontics, School of Dentistry, University of Zagreb, Zagreb, Croatia
}

Correspondence:

Dept. of Oral Medicine

School of Dentistry

University of Zagreb

Gunduliceva 5

10000 Zagreb,Croatia

borasvanja@yahoo.com

Boras VV, Savage NW, Brailo V, Lukac J, Lukac M, Alajbeg IZ. Salivary and serum levels of substance $P$, neurokinin $A$ and calcitonin gene related peptide in burning mouth syndrome. Med Oral Patol Oral Cir Bucal. 2010 May 1;15 (3):e427-31.

http://www.medicinaoral.com/medoralfree 01/v15i3/medoralv15i3p427.pdf

Received: 18/02/2009

Accepted: $12 / 09 / 2009$

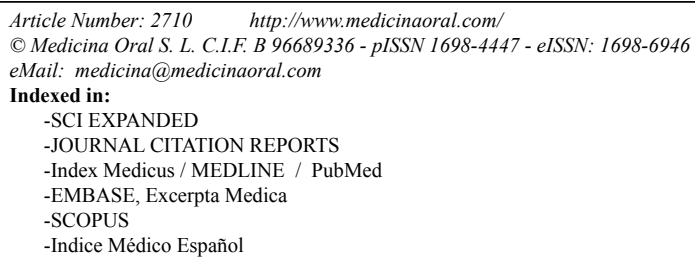

\begin{abstract}
Background: Burning mouth syndrome (BMS) is an enigmatic condition with the etiopathogenesis remaining largely obscure. However, a neuropathic basis for BMS continues to be an area of active clinical and research interest.

Aim: It is becoming increasingly evident that certain oral disorders may be modulated by imbalances in certain neuropeptides such as substance P (SP), neurokinin A (NKA) and calcitonin gene-related peptide (CGRP) therefore we measured SP, NKA and CGRP in the saliva and sera of BMS patients as well as controls.

Subjects and Methods: Salivary and serum SP, NKA and CGRP were determined in the 26 female patients with burning mouth syndrome (age range 51-78, mean 65.69 yrs), and in the 22 female controls (age range 24-82, mean 49.72 yrs). Serum and salivary SP, NKA, CGRP levels were determined by commercial competitive enzyme immunoassay kits. Statistical analysis was performed by use of descriptive statistics and analysis of variance.

Results and Conclusions: No significant differences in salivary SP, NKA and CGRP as well as serum SP and CGRP between BMS patients and controls could be found. However, significantly decreased serum neurokinin A $(\mathrm{p}<0.05)$ in BMS patients may reflect an inefficient dopaminergic system.
\end{abstract}

Key words: Substance $P$, neurokinin A, calcitonin gene-related peptide, burning mouth syndrome. 


\section{Introduction}

Burning Mouth Syndrome (BMS) is an oral sensory disorder diagnosed on the basis of history and the absence of any identifiable organic cause such as oral parafunctional habits, salivary gland dysfunction, local nerve trauma, menopausal disorders, diabetes, vitamin B group and iron deficiency (1). Notwithstanding the significant published literature on BMS, it continues to represent an enigmatic condition with the etiopathogenesis remaining largely obscure. It is poorly diagnosed and in many cases poorly managed and as such represents a source of frustration for both clinicians and patients. A neuropathic basis for BMS continues to be an area of active clinical and research interest. It is still, however, a matter of conjecture whether BMS is a centrally or peripherally induced pathologic condition. Svensson et al. (2) reported that BMS patients show an abnormal pre-pain perception intensity as well as disturbances in non-nociceptive and nociceptive thermal stimuli perception. Gao et al. (3) reported alterations in neuronal transmission together with a hyperalgesia and probable allodynia of the trigeminal nerve. Heckmann et al. (4) found disturbances in the mucosal neurovascular system. These reports together with taste changes and sensory/chemosensory disturbances (5-7) certainly support a neuropathic basis for BMS.

It is becoming increasingly evident that periodontitis and indeed other orofacial inflammatory disorders may be modulated by imbalances in certain neuropeptides. In periodontitis affected subjects, levels of both SP and NKA were significantly elevated in gingival crevicular fluid of disease-affected teeth compared with healthy sites. The role of neuropeptides in exerting a trophic effect on peripheral tissues is also worthy of mention. Such system has been proposed to act tonically so that normal sensory stimuli would produce a continuous outflow of sensory transmitters whose actions would maintain tissue integrity (8).

It is also important to note that nociceptive signals from periphery may be amplified or diminished during spinal processing, resulting in either central sensitization or desensitization, respectively. The stimulation of capsaicin-sensitive fibers (SP; CGRP) induce, on the one hand, an orthodromic noxious-sensory signal travelling toward cortical areas, and on the other hand, an axoaxon reflex of primary sensory fibers which antidromically releases transmitters from peripheral terminals evoking a number of changes in peripheral substrates (neurogenic inflammation, gland secretion, smooth muscle contraction). Therefore, it has been claimed that capsaicin-sensitive neurotransmitters are implicated in the pathophysiology of local reactions accompanying painful conditions. Despite the presence of degrading enzymes, saliva appears to be fluid which seemingly being close enough to peripheral receptors for sensory neuropeptides, can offer an image of the peptides release by sensory endings, roughly mirroring the central release pattern. In fact, capsaicin-sensitive neuropeptides (SP, CGRP) have unique property in that impulses may travel orthodromically or antidromically: therefore the transmitter may be released in both central and peripheral neural endings (9).

A number of neuropeptides exert a biologic effect that may play a role in the clinical presentation of BMS. Substance P (SP), neurokinin A (NKA) and calcitonin gene-related peptide (CGRP) for example all have active vasodilatory effects and the latter two play a direct role in pain. Furthermore they have attracted a considerable amount of attention in other pain syndromes including fibromyalgia and temporomandibular disorders (10). With the particular presentation scenario of BMS they may also be active in this condition. The aim of this project therefore was to evaluate salivary and serum SP, CGRP and NKA levels in the patients with BMS. The neuropeptides are released centrally and their levels might be reflected in serum whereas their local release from peripheral sensory nerves might be reflected in saliva. These data might provide more specific data upon neuropathology in BMS as being either central or peripheral.

\section{Materials and Methods}

This project received clearance from the Institutional Ethics Committee and patients provided written informed consent prior to commencement as required by Helsinki II. This study was realized during the years 2006 and 2007. The study group consisted of 26 female patients, age range 51-78, mean 65.69 years, with BMS. The diagnosis was made on the basis of a detailed clinical history and the exclusion of any local or systemic condition which may cause any type of oral dysaesthesia. They were not receiving specific treatment at the time of sampling. The control group consisted of 22 females with an age range of 24-82 and mean of 49.72 years. This was marginally younger than the BMS group but a requirement for inclusion was that individuals were not taking any medications for the previous month and this alone skewed the group somewhat.

All serum and saliva samples were collected between 10.00 and 13.00 to avoid circadian variation. Participants provided unstimulated whole saliva over a five minute period without swallowing. One $\mathrm{ml}$ of saliva was added to clean plastic containers with $3.9 \mathrm{mg}$ of ethylenediaminetetraacetate (EDTA) and $50 \mu \mathrm{l}$ of aprotinin $(70 \mathrm{mg} \mathrm{v} 50 \mathrm{ml}$ of sodium hypochlorate which is $500.000 \mathrm{KIE}$, Bayer AG, Germany) and the centrifuged at $1800 \mathrm{rpm}$ for 10 minutes.

Serum was collected from the antecubital veins into vacuum tubes containing $3.9 \mathrm{mg}$ of EDTA using standard venepuncture procedures (Venosafe evacuated 
blood collection tubes, Terumo, N.V., Leuven, Belgium). One $\mathrm{ml}$ of serum was added to tubes containing $50 \mu \mathrm{l}$ of aprotinin (70 $\mathrm{mg} \mathrm{v} 50 \mathrm{ml}$ of sodium hypochlorate which is 500.000 KIE, Bayer AG, Germany) and the mixture centrifuged at $1800 \mathrm{rpm}$ for ten minutes. Serum specimens were stored at $-20 \mathrm{C}$.

Serum and salivary neuropeptide levels were determined using commercial competitive enzyme immunoassay kits (Peninsula Laboratories Inc., Bachem Group, San Carlos, CA, USA) for human Substance P (Cat \# S-1180, detection range 0-10 ng/ml), Neurokinin A (Cat \# S-1280, detection range $0-25 \mathrm{ng} / \mathrm{ml}$ ) and $\alpha$-CGRP (Cat. \# S-1199, detection range $0-25 \mathrm{ng} / \mathrm{ml}$ ) with intra-assay and and inter-assay variabilities of $<5 \%$ and $<14 \%$ respectively. Following the manufacturer's instructions, $50 \mu \mathrm{l}$ aliquots of serum and saliva samples were dispensed into microplate wells and the target peptides were determined using a streptavidin-conjugated horseradish peroxidase with tetramethyl benzidine dihydrochloride as the substrate. The optical densities were read by the Dynatech MRX Microplate reader at $450 \mathrm{~nm}$. The results were expressed in $\mathrm{ng} / \mathrm{ml}$ of serum/saliva.

The statistical analysis was undertaken using the statistical software package SPSS 10.0 for Windows. Descriptive statistics were used and the normality of distribution was tested by one-way Kolmogorov-Smirnov test. Because the test showed a normal data distribution, subsequent analysis with parametric tests were undertaken. Analysis of variance (ANOVA) and pairwise comparisons (post hoc Scheffe test) were employed. $\mathrm{P}$-values less than 0.05 were considered as significant $(\mathrm{p}<0.05)$.

\section{Results}

No significant difference in unstimulated whole salivary flow rates was found between BMS patients and control subjects $(\mathrm{p}<0.05)$.

Table 1 presents the descriptive statistics of the specific neuropeptides in serum and saliva in the control and BMS groups respectively. Table 2 shows the results of Mann-Whitney analyses with a significant difference representing a depression in the BMS group for serum NKA values $(p<0.05)$. This depression does not however extend to any of the other neuropeptides in either serum or saliva at statistically significant levels. A review of Table 1, however, does show that whilst the depressed NKA levels do not extend to saliva, there is a trend showing an elevation of both SP and CGRP in both serum and saliva, as well as neurokinin A in saliva.

\section{Discussion}

The current study reviewed a limited number of BMS patients and, accepting the complexity of the condition described as BMS, the results are encouraging but at this early stage difficult to interpret with confidence. Accepting that the aetiology of BMS is currently unknown it seems likely that BMS conforms to the clinical pattern of a number of chronic pain conditions with the development of central nervous system pain processing abnormalities. This is the case for conditions as varied as fibromyalgia and temporomandibular joint disorders. In these conditions there seems to be a persistent nociceptive input from the peripheral tissues to the central nervous system leading to neuroplastic changes and a resultant central sensitization and pain (10). The immediate downstream outcome is that minimal peripheral stimulation only is required to maintain the chronic pain syndrome. Patients also experience involvement of previously uninvolved sites with the production of pain by very low grade stimulation. This is directly transferrable to the clinical presentation of BMS where the anterior dorsum and anterolateral tongue margins are most commonly involved with extension to other sites as a subsequent event or recognition only as a secondary low grade phenomenon on clinical questioning.

Decreased levels of dopamine likely contribute to the painful symptoms that frequently occur in Parkinson's disease. Moreover, abnormalities in dopaminergic neurotransmission have been objectively demonstrated in painful clinical conditions, including burning mouth syndrome, fibromyalgia and restless leg syndrome. Likewise, reduced levels of NKA in the serum of patients with BMS may reflect a deficiency in the dopaminergic system and some commentators have suggested that such a deficient dopaminergic influence might actually be causative in BMS (11). Electrophysiological examinations in BMS patients indicate an abnormal blink reflex which is under control of dopaminergic inhibitory control through the basal ganglia connection with the facial motor nuclei (12). An abnormal blink reflex is also a common finding in extra-pyramidal disorders such as Parkinson's disease and facial dyskinesias $(13,14)$ where it may be caused by a deficient dopaminergic influence on the brainstem nuclei. Further supportive evidence is found in a decreased dopaminergic inhibition in BMS patients which primarily affects trigeminal nerve nociception and leads to the loss of sensory inhibition (15). A generalized and possibly multi-level abnormality in the processing of somatosensory information has also been found in BMS patients and this appears to be the result of peripheral neurogenic mechanism pathology (16). It is also well known that tachykinins such as SP and others are crucial for the functioning of dopaminergic neurons.

Furthermore, decreased levels of NKA have been linked with number of drugs which act directly on receptors located on airway sensory nerves and inhibit neuropeptide release, for example opioids and local anesthetics (17). However, we couldnt find any association between 
Table 1. Descriptive statistics of neurokinin A (NKA), substance P (SP) and calcitonin-gene-related peptide (CGRP) in serum and saliva of the control and burning mouth syndrome group.

\begin{tabular}{|c|c|c|c|c|c|c|c|c|c|c|c|c|}
\hline & \multicolumn{2}{|c|}{ NKA serum } & \multicolumn{2}{|c|}{ NKA saliva } & \multicolumn{2}{|c|}{ SP serum } & \multicolumn{2}{|c|}{ SP saliva } & \multicolumn{2}{|c|}{ CGRP serum } & \multicolumn{2}{|c|}{ CGRP saliva } \\
\hline & CG & BMS & CG & BMS & CG & BMS & CG & BMS & CG & BMS & $\mathrm{CG}$ & BMS \\
\hline N Valid & 22 & 26 & 22 & 26 & 22 & 26 & 22 & 26 & 22 & 26 & 22 & 26 \\
\hline Mean & 0,234 & 0,193 & 0,101 & 0,111 & 0,151 & 0,192 & 0,095 & 0,164 & 0,260 & 0,350 & 0,150 & 0,206 \\
\hline $\begin{array}{l}\text { Std. Error } \\
\text { of Mean }\end{array}$ & 0,014 & 0,011 & 0,025 & 0,013 & 0,015 & 0,023 & 0,026 & 0,040 & 0,043 & 0,063 & 0,028 & 0,036 \\
\hline Median & 0,221 & 0,184 & 0,085 & 0,085 & 0,159 & 0,171 & 0,074 & 0,125 & 0,186 & 0,225 & 0,105 & 0,128 \\
\hline $\begin{array}{l}\text { Std. Devia- } \\
\text { tion }\end{array}$ & 0,064 & 0,057 & 0,116 & 0,065 & 0,071 & 0,119 & 0,122 & 0,206 & 0,200 & 0,322 & 0,131 & 0,183 \\
\hline Variance & 0,004 & 0,003 & 0,013 & 0,004 & 0,005 & 0,014 & 0,015 & 0,043 & 0,040 & 0,103 & 0,017 & 0,033 \\
\hline Minimum & 0,149 & 0,126 & 0,001 & 0,033 & 0,040 & 0,054 & 0,001 & 0,011 & 0,086 & 0,050 & 0,012 & 0,027 \\
\hline Maximum & 0,386 & 0,383 & 0,590 & 0,299 & 0,306 & 0,422 & 0,590 & 1,078 & 0,772 & 1,265 & 0,498 & 0,634 \\
\hline
\end{tabular}

NKA-neurokinin, SP-substance P, CGRP-calcitonin-gene-related peptide, CG-control group,BMS-patients with burning mouth syndrome.

Table 2. Mann-Whitney $U$ test between BMS patients and the control group regarding neurokinin A (NKA), substance P (SP) and calcitonin-gene-related peptide (CGRP) in serum and saliva.

\begin{tabular}{|l|c|c|c|c|c|c|}
\hline \multicolumn{7}{|c|}{ Mann-Whitney U test between patients with burning mouth syndrome and } \\
control subjects \\
\hline & $\begin{array}{c}\text { NKA } \\
\text { serum }\end{array}$ & $\begin{array}{c}\text { NKA } \\
\text { saliva }\end{array}$ & $\begin{array}{c}\text { SP } \\
\text { serum }\end{array}$ & $\begin{array}{c}\text { SP } \\
\text { saliva }\end{array}$ & $\begin{array}{c}\text { CGRP } \\
\text { serum }\end{array}$ & $\begin{array}{c}\text { CGRP } \\
\text { saliva }\end{array}$ \\
\hline Mann-Whitney U & 165,5 & 226 & 247,5 & 191,5 & 254 & 241 \\
\hline P value & $0,013^{*}$ & 0,214 & 0,426 & 0,05 & 0,508 & 0,352 \\
\hline
\end{tabular}

medications our BMS patients used and decreased serum neurokinin A levels.

The salivary elevation of all investigated tachykinins although not being significant is interesting from two aspects. Salivary neuropeptides decrease in stimulated saliva (18) and so the current elevation does represent a real increase given the unstimulated saliva collection method. Secondly this increase might reflect neurogenic inflammation which might be causative to BMS. SP and NKA exert a wide variety of biological activities and are intimately linked with neurogenic inflammation, which has been shown to have a dose-dependent relationship with the levels of SP and/or NKA. One of the reasons why increased levels of salivary tachykinins did not reach significance might be mucosal atrophy which was found in $70 \%$ of BMS patients (19) which is known to influence the levels of certain salivary constituents i.e. (their leakage into saliva).

However, our results are not in concordance with the results of Zidverc-Trajkovic et al. (20) who reported that patients with BMS had decreased salivary CGRP levels. Furthermore, the same authors (20) conclude that this decrease is a consequence of trigeminal nerve degene-ration.
In conclusion the current investigation shows a strong trend to elevation of neuropeptides in both saliva and serum with the single exception of serum NKA. The implication is that this may play a direct role in the progression of BMS and explain comorbidities reported with this condition. Further investigation is required but the authors are encouraged by the present findings and feel that the likely relationship of BMS with other chronic pain syndromes may provide a direction for future management.

\section{References}

1. Scala A, Checchi L, Montevecchi M, Marini I, Giamberardino MA. Update on burning mouth syndrome: overview and patient management. Crit Rev Oral Biol Med. 2003;14:275-91.

2. Svensson P, Bjerring P, Arendt-Nielsen L, Kaaber S. Sensory and pain thresholds to orofacial argon laser stimulation in patients with chronic burning mouth syndrome. Clin J Pain. 1993;9:207-15.

3. Gao S, Wang Y, Wang Z. Assessment of trigeminal somatosensory evoked potentials in burning mouth syndrome. Chin J Dent Res. 2000;3:40-6.

4. Heckmann SM, Heckmann JG, HiIz MJ, Popp M, Marthol H, Neundörfer B, et al. Oral mucosal blood flow in patients with burning mouth syndrome. Pain. 2001;90:281-6.

5. Grushka M, Sessle BJ, Howley TP. Psychophysical assessment of 
tactile, pain and thermal sensory functions in burning mouth syndrome. Pain. 1987;28:169-84.

6. Femiano F, Lanza A, Buonaiuto C, Gombos F, Cirillo N. Burning mouth disorder (BMD) and taste: a hypothesis. Med Oral Patol Oral Cir Bucal. 2008;13:E470-4.

7. Grushka M, Sessle BJ. Burning mouth syndrome. Dent Clin North Am. 1991;35:171-84.

8. Lundy FT, Linden GJ. Neuropeptides and neurogenic mechanisms in oral and periodontal inflammation. Crit Rev Oral Biol Med. 2004;15:82-98.

9. Nicolodi M, Del Bianco E. Sensory neuropeptides (substance P, calcitonin gene-related peptide) and vasoactive intestinal polypeptide in human saliva: their pattern in migraine and cluster headache. Cephalalgia. 1990;10:39-50.

10. Staud R, Spaeth M. Psychophysical and neurochemical abnormalities of pain processing in fibromyalgia. CNS Spectr. 2008;13:12-7.

11. Wood PB. Role of central dopamine in pain and analgesia. Expert Rev Neurother. 2008;8:781-97.

12. Jääskeläinen SK, Forssell H, Tenovuo O. Abnormalities of the blink reflex in burning mouth syndrome. Pain. 1997;73:455-60.

13. Kimura J. Disorder of interneurons in Parkinsonism. The orbicularis oculi reflex to paired stimuli. Brain. 1973;96:87-96.

14. Berardelli A, Rothwell JC, Day BL, Marsden CD. Pathophysiology of blepharospasm and oromandibular dystonia. Brain. 1985;108:593-608

15. Jääskeläinen SK, Rinne JO, Forssell H, Tenovuo O, Kaasinen V, Sonninen P, et al. Role of the dopaminergic system in chronic pain -- a fluorodopa-PET study. Pain. 2001;90:257-60.

16. Forssell H, Jääskeläinen S, Tenovuo O, Hinkka S. Sensory dysfunction in burning mouth syndrome. Pain. 2002;99:41-7.

17. Pérez Fontan JJ. On lung nerves and neurogenic injury. Ann Med. 2002;34:226-40

18. Dawidson I, Blom M, Lundeberg T, Theodorsson E, AngmarMånsson B. Neuropeptides in the saliva of healthy subjects. Life Sci. 1997;60:269-78.

19. Lauritano D, Spadari F, Formaglio F, Zambellini Artini M, Salvato $A$. Etiopathogenic, clinical-diagnostic and therapeutic aspects of the burning mouth syndrome. Research and treatment protocols in a patient group. Minerva Stomatol. 1998;47:239-51.

20. Zidverc-Trajkovic J, Stanimirovic D, Obrenovic R, Tajti J, Vécsei L, Gardi J, et al. Calcitonin gene-related peptide levels in saliva of patients with burning mouth syndrome. J Oral Pathol Med. 2009;38:29-3. 